\title{
Urogynecology Section of the Polish Society of Gynecologists and Obstetricians Guidelines on the management of recurrent pelvic organ prolapse
}

\author{
Wlodzimierz Baranowski ${ }^{1}$, Klaudia Stangel-Wojcikiewicz ${ }^{2}\left(\mathbb{D}\right.$, Magdalena E. Grzybowska ${ }^{3}$,

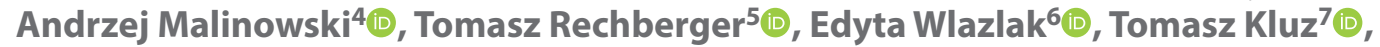

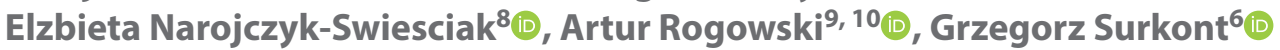 \\ 'Department of Gynecology, Gynecological Oncology, Military Medical Institute, Warsaw, Poland \\ ${ }^{2}$ Department of Gynecology and Oncology, Jagiellonian University Medical College, Cracow, Poland \\ ${ }^{3}$ Department of Gynecology, Gynecological Oncology and Gynecological Endocrinology, Medical University of Gdansk, Poland \\ ${ }^{4}$ Department of Surgical and Endoscopic Gynaecology, Medical University in Lodz, Poland \\ ${ }^{5} 2^{\text {nd }}$ Department of Gynecology, Medical University of Lublin, Poland \\ ${ }^{6}$ Department of Operative Gynecology and Gynecological Oncology, I Department of Gynecology and Obstetrics, \\ Medical University of Lodz, Poland \\ ${ }^{7}$ Department of Gynecology, Gynecology Oncology and Obstetrics, Institute of Medical Sciences, \\ Medical College of Rzeszow University, Poland \\ ${ }^{8} 2^{\text {nd }}$ Department of Obstetrics and Gynecology, The Center of Postgraduate Medical Education, Warsaw, Poland \\ ${ }^{9}$ Department of Gynecology, "Inflancka" Specialist Hospital, Warsaw, Poland \\ ${ }^{10}$ Collegium Medicum, Cardinal Stefan Wyszynski University, Warsaw, Poland
}

\begin{abstract}
Objectives: The aim of the publication was to present the Guideline of the Urogynecology Section of the Polish Society of Gynecologists and Obstetricians (PSGO) for the management of recurrent pelvic organ prolapse, based on the available literature, expert knowledge and opinion, as well as everyday practice.

Material and methods: In 2005, 2006 and 2010, the panel of PSGO experts published guidelines for the diagnosis and treatment of patients with lower urinary tract symptoms (LUTS). This publication presents an update of those recommendations and concerns recurrent POP treatment.

Main conclusion: The analysis of data revealed that sacrocolpopexy with the use of commercial sets or polypropylene hernia mesh is the method of choice for the surgical repair of recurrent vaginal vault prolapse. However, a significantly higher risk of surgical and postoperative complications after sacrocolpopexy, as compared to vaginal surgeries, should be considered when making treatment decisions. In other types of recurrent POP, the choice of surgery method should be tailored to the individual needs of each patient and may depend on the medical center.
\end{abstract}

Key words: pelvic organ prolapse; recurrence; reoperation

Ginekologia Polska 2022; 93, 2: 173-176

\section{INTRODUCTION}

1. Types of pelvic organ prolapse (POP) recurrence:

- POP recurrence within the previously operated site,

- POP or progression of a pre-existing prolapse within the non-operated compartment (e.g., surgical correction of anterior vaginal wall prolapse and postoperative symptomatic posterior vaginal wall prolapse),

- POP recurrence within the previously operated compartment, but in a different anatomic location (e.g., surgical repair of the central defect of the anterior vaginal wall and postoperative presentation of the 
lateral defect of the anterior vaginal wall - the so-called 'masked defect').

2. Objective assessment of the surgical success:

- anatomic assessment (typically using the POP-Q scale),

- reoperation rate for same site or new site recurrent prolapse,

- reoperation rate due to complications (mesh exposure, pain, different types of postoperative voiding dysfunctions - urine retention, urinary incontinence, overactive bladder).

3. Subjective (patient-reported) assessment of the surgical success:

- subjective assessment of the postoperative success by the patient (e.g. using the Patient Global Impression of Improvement (PGI-I) scale),

- validated quality of life (QoL) questionnaires after POP surgery [1].

4. Reoperation rate:

- reoperation rate after traditional (native tissue) repair $-16 / 1000$,

- reoperation rate after implant surgery $-7 / 1000$, $(\mathrm{RR}=0.44 ; \mathrm{Cl} 0.24-0.81)$,

- POP recurrence assessed objectively (POPQ > 2): traditional (native tissue) $-41 \%$, synthetic implants - 10.1-18.7\% (RR = 0.34; Cl 0.25-0.46) [1--4]

\section{Objectives}

The aim of the Urogynecology Section of the Polish Society of Gynecologists and Obstetricians (PSGO) was to develop this Guideline for the management of recurrent pelvic organ prolapse, based on the available literature, expert knowledge and opinion as well as everyday practice.

\section{Material and methods}

In 2005, 2006 and 2010, the panel of PSGO experts developed guidelines for the diagnosis and treatment of patients with lower urinary tract symptoms (LUTS). This publication presents an update of those recommendations and concerns recurrent POP treatment.

\section{RECOMMENDATIONS}

\section{Recommendations on the management of POP} recurrence in the anterior compartment

Recurrence after traditional (native) surgery in the anterior compartment: consider using prosthetic materials [90-95\% of total anatomic success; at two years of follow-up $53 \%$ of the patients presented with POP-Q $\leq 2$ and $42 \%$ with POP- Q $\leq 1$ as compared to only $55 \%$ anatomic success using native tissue reoperation] $[5,6]$. Recent findings of a retrospective study conducted in Australia among 196 patients, also demonstrated better anatomic outcome (point $\mathrm{Ba}=0 \mathrm{~cm}$ of the $\mathrm{POP}-\mathrm{Q}$ ) after the repair of the recurrent anterior vaginal wall defect using prosthetic materials as compared to native tissue reoperation $-25 \%$ recurrence in the TVM group vs $>40 \%$ in the classic reoperation group [2]. Also, the patients from the implant group reported a significantly higher subjective improvement in the quality of life ( $88 \%$ vs $66 \%, p<0.01)$. The risk for yet another reoperation was significantly lower in the implant group $(7.4 \%$ vs $23.9 \%, p<0.01)$, but the high rate of mesh exposure (15\%) and the related need for reoperation (9\%), raise serious concern regarding the use of the prosthetic material method, despite its greater effectiveness [5-12].

\section{Conclusions}

The use of prosthetic materials in reoperations due to recurrent prolapse of the anterior vaginal wall results in better anatomic and functional outcome, however mesh exposure and postoperative pain syndrome, most often associated with excessive retraction of the synthetic material, constitute a significant issue. Reoperations with the use of prosthetic material should be conducted by a team with extensive experience performing urogynecological surgeries. We still wait for the results of studies using lighter weight new-generation implants.

\section{Recommendations on the management of POP recurrence in the posterior compartment}

According to the $2017 \mathrm{ICl}$ guidelines, prosthetic materials (biologic and synthetic) may be used in the posterior compartment in the rectovaginal space for primary surgery and for recurrent POP in that compartment [1]. As far as reoperation due to recurrent prolapse in the previously operated posterior compartment is concerned, the literature offers only one study comparing the effectiveness of the traditional versus synthetic implant repairs. Those authors found that the use of synthetic prosthetic material resulted in significantly better anatomic outcome as compared to native tissue repair (anatomic success: $92.5 \%$ vs $59.1 \% ; p=0.01$, subjective feeling of prolapse only in: $7.5 \%$ vs $24.1 \% ; p=0.02$, need for yet another reoperation: $7.5 \%$ vs $19.5 \% ; p=0.08)$. An analysis of the composite outcomes also confirmed the superiority of prosthetic material repairs (56.6\% vs $23.0 \%$; $p<0.01$ ), however implant-related complications mesh exposures continue to be a problem - mesh removal surgery was necessary in $15.1 \%$ of the patients after synthetic implant repair $[2,3,13,14]$.

\section{Conclusions}

The use of synthetic prosthetic materials increases the chances for permanent recovery in patients reoperated due to recurrent prolapse in the posterior compartment. High 
rate of mesh exposures which require surgical management remains an unresolved issue. Therefore, reoperation without using synthetic material may be another option in selected cases.

\section{Recommendations on the management of POP recurrence in the central compartment}

A. Reoperations due to recurrent POP after primary repair in the central compartment in women with preserved uterus present a serious challenge to the decision-making and surgical processes. We must consider two different group of patients:

- recurrences after traditional vaginal surgeries - modified Manchester repair (Fothergill operation), sacrospinous ligament (SSLF), uterosacral ligament suspension (USLS), medial closure of the vaginal walls.

- recurrences after vaginal surgeries using synthetic prosthetic materials (commercial sets for sacrospinous ligament suspension from the anterior or the posterior approach)

B. Reoperations due to recurrent vaginal vault prolapse after hysterectomy (abdominal or vaginal).

The literature offers limited and inconclusive data on the techniques of reoperation for recurrent prolapse in the central compartment. However, after critical analysis of the available data, it seems safe to conclude that in patients with preserved uterus/cervix and after failed native tissue repair (Manchester-Fothergill, SSLF, USLS, median closure of the vaginal walls), a transvaginal repair surgery using synthetic materials may be considered in case of a two-compartment defect (central and anterior or central and posterior). The use of second generation meshes with sacrospinous ligament fixation in the treatment of the central compartment disorders may be associated with a better anatomic effect as compared to the first-generation implants.

In case of defects in three compartments, abdominal surgery (classic, laparoscopic, robotic) is often recommended - hysterosacropexy, cervico-sacropexy. Such management is also often recommended in patients with recurrent prolapse who underwent primary transvaginal surgery with synthetic materials.

In patients with vaginal vault prolapse after hysterectomy, regardless of whether the prolapse is primary or after vaginal repair surgery (native or with prosthetic materials), classical or laparoscopic sacrocolpopexy are often recommended. The risk for POP recurrence in the central compartment is higher in patients operated from the transvaginal as compared to the transabdominal approach (RR 1.89; $95 \% \mathrm{Cl} 1.33$ to 2.70 ) - in absolute numbers: $41 \%$ vs $23 \%$ [20]. A meta-analysis demonstrated sacrocolpopexy to be more effective in terms of anatomic success as compared to transvaginal reoperations but is associated with the risk for gastrointestinal (2.7\%) and implant-related (4.2\%) complications, as well as thromboembolic events (0.6\%) [16-19]. Significantly higher invasiveness of sacrocolpopexy, and the related risk for surgical and postoperative complications, should be considered when making therapeutic decisions. Importantly, objective data on reoperation techniques for recurrent prolapse in the central compartment remain limited and inconclusive.

\section{Conclusions}

The analysis demonstrated that sacrocolpopexy, with the use of commercial sets or polypropylene hernia mesh, should be recommended as the procedure of choice for recurrent vaginal vault prolapse. However, while making surgical decisions, one should consider a significantly higher risk for peri- and post-operative complications after sacrocolpopexy as compared to the vaginal approach. Therefore, the choice of surgery should be tailored to the individual needs of every patient and may vary between medical centers.

\section{GUIDELINE SUMMARY}

In accordance with the $2017 \mathrm{ICl}$ recommendations and the "Consensus of the 2nd IUGA Grafts Roundtable", the use of synthetic prosthetic materials is justified in all cases of recurrent prolapse, regardless of the POP compartment. At the same time, it is also allowed to perform these surgeries without using synthetic materials. The choice of surgery should be strongly personalized. Among others, special attention should be paid to the risk for complications.

\section{Conflict of interest}

All authors declare no conflict of interest.

\section{REFERENCES}

1. Maher C, Baessler K, Barber M. Pelvic Organ Prolapse Surgery. In: Abrams $\mathrm{P}$, Cardozo L, Wagg A, Wein A. ed. Incontinence 6th edition . ICUD ICS 2017: 1855-1992.

2. Ow L, Lim $Y$, Dwyer $P$, et al. Native tissue repair or transvaginal mesh for recurrent vaginal prolapse: what are the long-term outcomes? Int Urogynecol J. 2016; 27(9): 1313-1320, doi: 10.1007/s00192-016-3069-6.

3. Gutman RE, Nosti PA, Sokol Al, et al. Three-year outcomes of vaginal mesh for prolapse: a randomized controlled trial. Obstet Gynecol. 2013; 122(4): 770-777, doi: 10.1097/AOG.0b013e3182a49dac, indexed in Pubmed: 24084533.

4. Iglesia CB, Sokol Al, Sokol ER, et al. Vaginal mesh for prolapse: a randomized controlled trial. Obstet Gynecol. 2010; 116(2 Pt 1): 293-303, doi: 10.1097/AOG.0b013e3181e7d7f8, indexed in Pubmed: 20664388.

5. Fayyad A, North C, Reid F, et al. Prospective study of anterior transobturator mesh kit (Prolift ${ }^{T M}$ ) for the management of recurrent anterior vaginal wall prolapse. Int Urogynecol J. 2010; 22(2): 157-163, doi: 10.1007/s00192-010-1260-8.

6. Withagen M, Milani A, Boon Jd, et al. Trocar-Guided Mesh Compared With Conventional Vaginal Repair in Recurrent Prolapse. Obstet Gynecol. 2011; 117(2): 242-250, doi: 10.1097/aog.0b013e318203e6a5.

7. Rudnicki M, Laurikainen E, Pogosean R, et al. A 3-year follow-up after anterior colporrhaphy compared with collagen-coated transvaginal mesh for anterior vaginal wall prolapse: a randomised controlled trial. $\mathrm{Br}$ J Obstet Gynaecol. 2015; 123(1): 136-142, doi: 10.1111/1471-0528.13628. 
8. Dias MM, de A Castro R, Bortolini MA, et al. Two-years results of native tissue versus vaginal mesh repair in the treatment of anterior prolapse according to different success criteria: A randomized controlled trial. Neurourol Urodyn. 2016; 35(4): 509-514, doi: 10.1002/nau.22740, indexed in Pubmed: 25820682.

9. Tamanini JT, de Oliveira Souza Castro RC, Tamanini JM, et al. A prospective, randomized, controlled trial of the treatment of anterior vaginal wall prolapse: medium term followup. J Urol. 2015; 193(4): 1298-1304, doi: 10.1016/j.juro.2014.10.003, indexed in Pubmed: 25305357.

10. de Tayrac R, Cornille A, Eglin G, et al. Comparison between trans-obturator trans-vaginal mesh and traditional anterior colporrhaphy in the treatment of anterior vaginal wall prolapse: results of a French RCT. Int Urogynecol J. 2013;24(10): 1651-1661, doi: 10.1007/s00192-013-2075-1, indexed in Pubmed: 23512113.

11. Nieminen $K$, Hiltunen $R$, Takala $T$, et al. Outcomes after anterior vaginal wall repair with mesh: a randomized, controlled trial with a 3 year follow-up. Am J Obstet Gynecol. 2010; 203(3): 235.e1-235.e8, doi: 10.1016/j.ajog.2010.03.030, indexed in Pubmed: 20494332.

12. Vollebregt $\mathrm{A}$, Vaart $\mathrm{CHv}$. Primary surgical repair of anterior vaginal prolapse: a randomised trial comparing anatomical and functional outcome between anterior colporrhaphy and trocar-guided transobturator anterior mesh. BJOG: An International Journal of Obstetrics \& Gynaecology. 2012; 119(9): 1151-1152, doi: 10.1111/j.1471-0528.2012.03372.x.

13. Maher C, Feiner B, Baessler K, et al. Transvaginal mesh or grafts compared with native tissue repair for vaginal prolapse. Cochrane Database Syst Rev. 2016; 2: CD012079, doi: 10.1002/14651858.CD012079, indexed in Pubmed: 26858090.

14. Dos Reis Brandão da Silveira S, Haddad JM, de Jármy-Di Bella Zl, et al. Multicenter, randomized trial comparing native vaginal tissue repair and synthetic mesh repair for genital prolapse surgical treatment. Int Urogynecol J. 2015; 26(3): 335-342, doi: 10.1007/s00192-014-2501-z, indexed in Pubmed: 25199496.

15. Altman D, Vayrynen T, Engh ME, et al. Anterior colporraphy versus transvaginal mesh for pelvic organ prolapse. N Eng J Med. 2011;364: 1826-36.

16. Siddiqui NY, Grimes CL, Casiano ER, et al. Society of Gynecologic Surgeons Systematic Review Group. Mesh sacrocolpopexy compared with native tissue vaginal repair: a systematic review and meta-analysis. $\mathrm{Ob}$ stet Gynecol. 2015; 125(1):44-55, doi: 10.1097/AOG.0000000000000570, indexed in Pubmed: 25560102.

17. Lamblin G, Van-Nieuwenhuyse A, Chabert $P$, et al. A randomized controlled trial comparing anatomical and functional outcome between vaginal colposuspension and transvaginal mesh. Int Urogynecol J. 2014; 25(7): 961-970, doi: 10.1007/s00192-014-2344-7, indexed in Pubmed: 24573358.

18. Maher C, Feiner B, Baessler $\mathrm{K}$, et al. Surgery for women with apical vaginal prolapse. Cochrane Database Syst Rev. 2016, doi: 10.1002/14651858.cd012376.

19. Barber $M D$, Brubaker $L$, Burgio $K L$, et al. Eunice Kennedy Shriver National Institute of Child Health and Human Development Pelvic Floor Disorders Network. Comparison of 2 transvaginal surgical approaches and perioperative behavioral therapy for apical vaginal prolapse: the OPTIMAL randomized trial. JAMA. 2014; 311(10): 1023-1034, doi: 10.1001/jama.2014.1719, indexed in Pubmed: 24618964.

20. Davila GW, Baessler K, Cosson M, et al. Selection of patients in whom vaginal graft use may be appropriate. Consensus of the 2nd IUGA Grafts Roundtable: optimizing safety and appropriateness of graft use in transvaginal pelvic reconstructive surgery. Int Urogynecol J. 2012; 23 Suppl 1: S7-14, doi: 10.1007/s00192-012-1677-3, indexed in Pubmed: 22395288 